\title{
Fuji-san Classification Predicts Fibrinogen Concentration after Allogeneic Blood Transfusion but not after Hemodilutional Autologous Blood Transfusion in Cardiac surgery with Cardiopulmonary Bypass.
}

\author{
Hiroshi SAKAMOTO, Syunsuke EDAKUBO, Yoshihide MIURA, Katsumi HARASAWA \\ Department of Anesthesia, Hokkaido Ohno Memorial Hospital, Sapporo, 0630052, Japan
}

Background and Goal of Study: We calculated estimated fibrinogen concentration based on the formula in Fuji-san classification and compared measured on-site fibrinogen concentration by CG02N (A \& $\mathrm{T}$ Corporation, Yokohama, Japan) to optimize fibrinogen replacement therapy.

Patients and Methods: After obtaining IRB approval and informed consent, 237 patients undergoing scheduled cardiac surgery with cardiopulmonary bypass (CPB) were enrolled in this study. Among them, recent 16 patients received surgery with hemodilutional autologous blood transfusion (HAT group). In other 221 patients, hemodilutional autologous blood transfusion was not performed (Control group). In HAT group, the amount of blood to be removed was calculated with the following formula: $80 \times$ body weight $(\mathrm{kg}) \times(1-$ $27 /$ Hct (\%)) with the utmost $800 \mathrm{ml}$. On site fibrinogen concentration (FIB) was measured with CG02N at the arrival in OR, heparin reversal and conclusion of surgery and blood loss during surgery were recorded. Fibrinogen concentration at the conclusion of surgery was estimated with the following formula according to Fuji-san classification: 187.5 $\times(1-\exp (-(16000 \times \mathrm{FFP}) /(70 \times \mathrm{BW} \times(100-\mathrm{Hct})))+\mathrm{FIB} \times \exp (-(160$ $\times \mathrm{FFP}) /(70 \times \mathrm{BW}))$. In this formula, FFP is fresh frozen plasma (units) transfused after heparin reversal, BW is patient's body weight $(\mathrm{kg})$ and FIB is fibrinogen concentration $(\mathrm{mg} / \mathrm{dl})$ at heparin reversal. In HAT group, FIB content of autologous blood is calculated and every $250 \mathrm{mg}$ of FIB is considered equivalent to 1 unit of FFP. Allogeneic FFP in units and autologous blood in equivalent FFP units were applied to Fuji-san formula to estimate FIB in HAT group. Data were compared using paired and unpaired t-test. A $p$-value $<0.05$ was considered statistically significant.

Results and Discussion: Patients' demographic data showed no significant difference except preoperative hematocrit (Table 1.and 2.). Right after heparin reversal, measured FIB in HAT group was significantly lower than that in control group. But calculated FIB, which equals the sum of measured FIB and fibrinogen in collected autologous blood per circulating blood volume (4\% of BW), showed no significant difference and correlation coefficient between measured and calculated FIB was 0.838 in HAT group (Table 3.). It may mean the difference of measured FIB between HAT and Control groups is due to normovolemic hemodilutional autologous blood collection. In Control group, measured FIB and estimated FIB showed no significant difference at the conclusion of surgery. In HAT group, estimated FIB was significantly lower than measured FIB at the conclusion of surgery. The calculated FIB is a good predictor of future FIB after FFP transfusion in Control group but not in HAT group (Table 4.). We calculated FIB by adding FIB estimated by FFP based on Fuji-san classification formula and FIB amount in autologous blood. Measured FIB and calculated FIB showed no significant difference at the conclusion of surgery (Table 5). According to result from HAT group, the hemostatic effect of normovolemic hemodilution and fresh autologous blood should be different from that of allogeneic FFP and estimation of FIB should be also different.

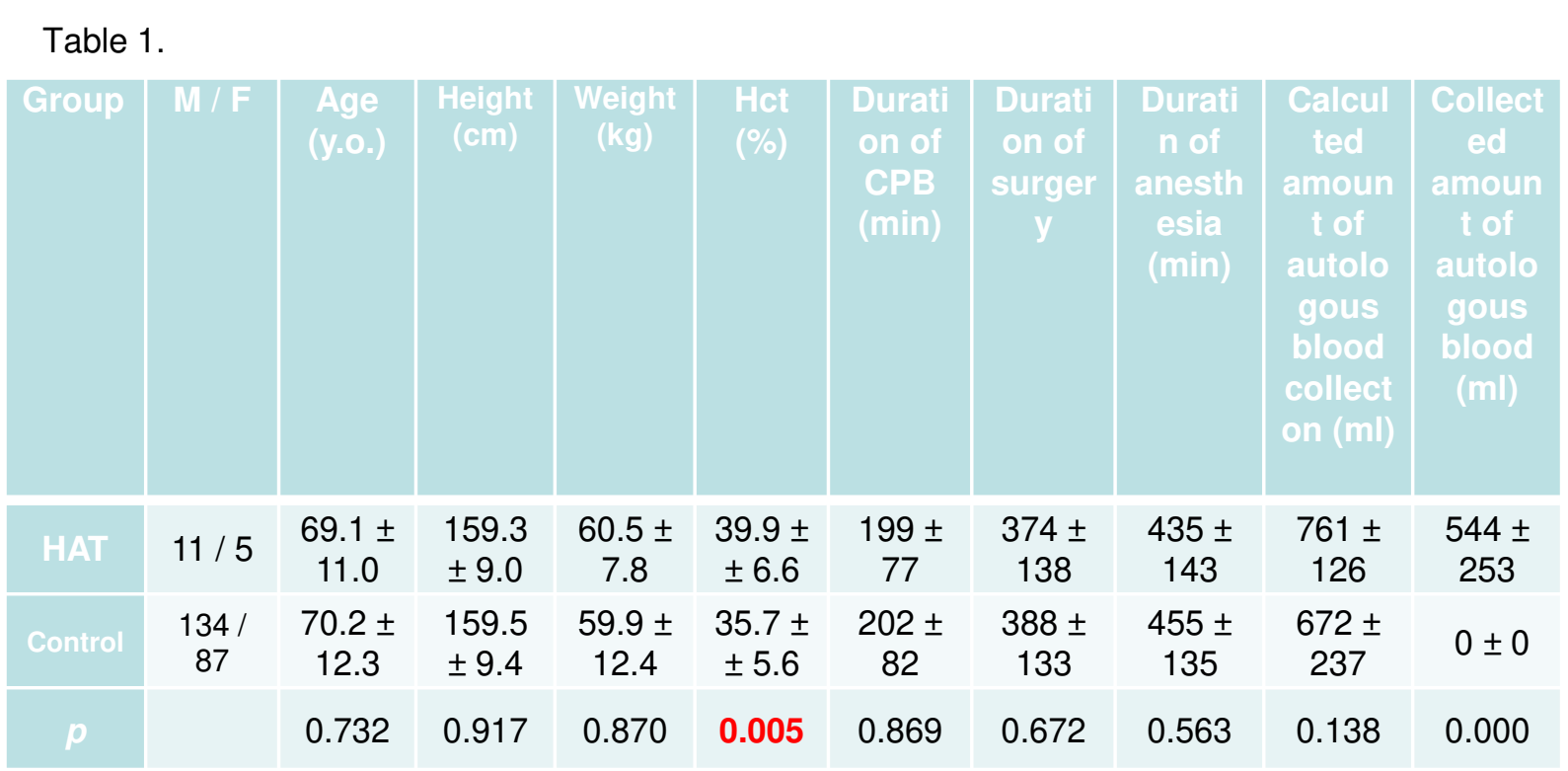

Data are shown in mean \pm S.D

Table 2.

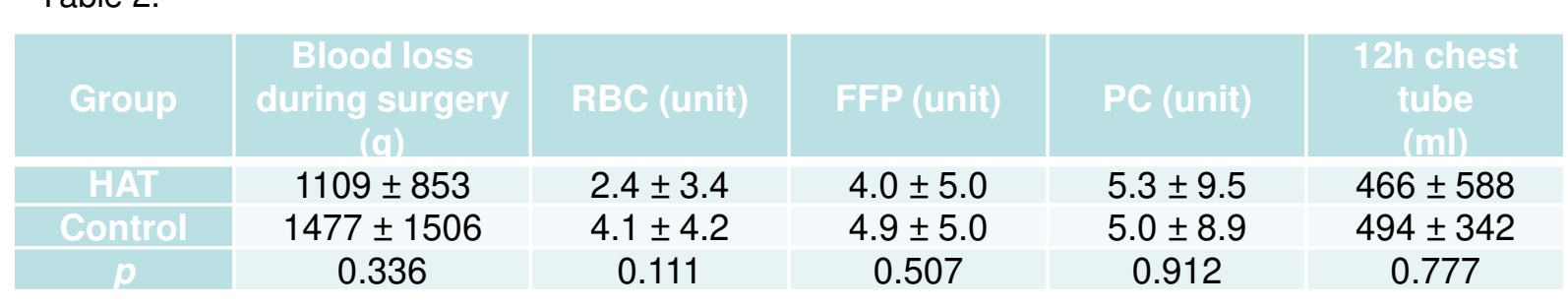

Data are shown in mean \pm S.D.

Table 3.

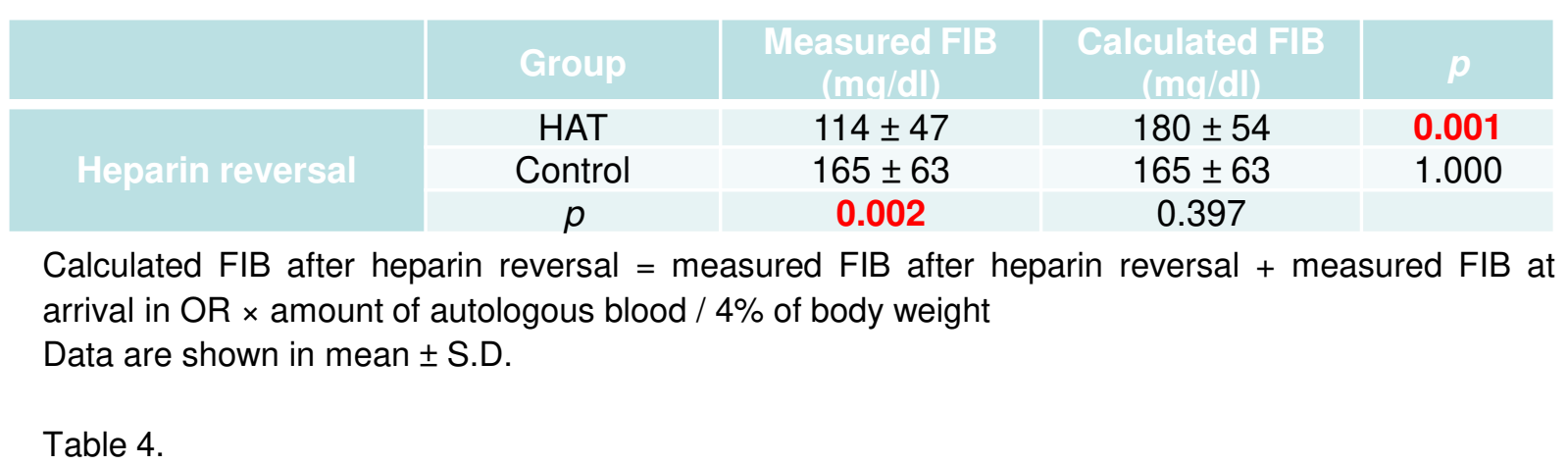

Table 4 .

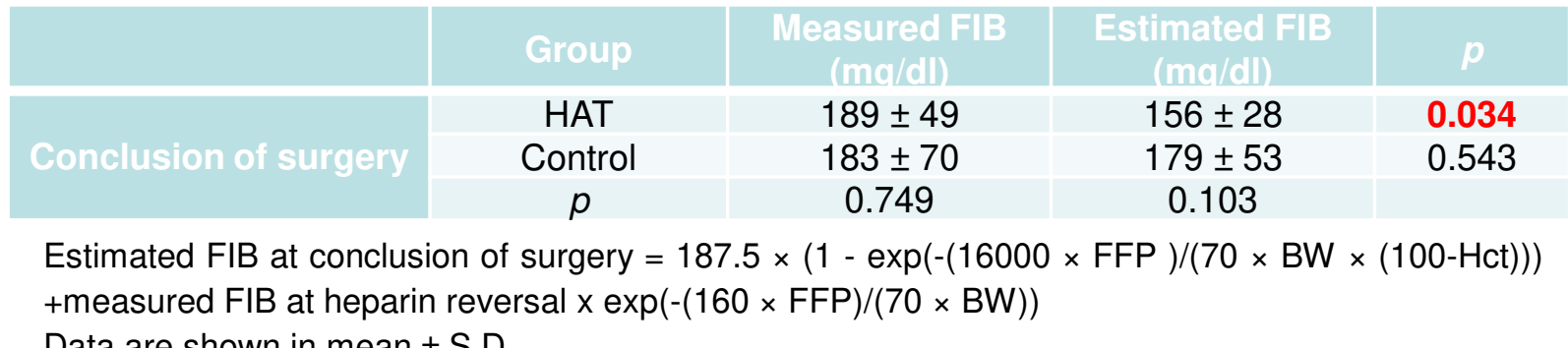
+measured FIB at heparin reversal $\times \exp (-(160 \times \mathrm{FFP}) /(70 \times \mathrm{BW}))$ Data are shown in mean \pm S.D.

$$
\text { Table } 5 .
$$

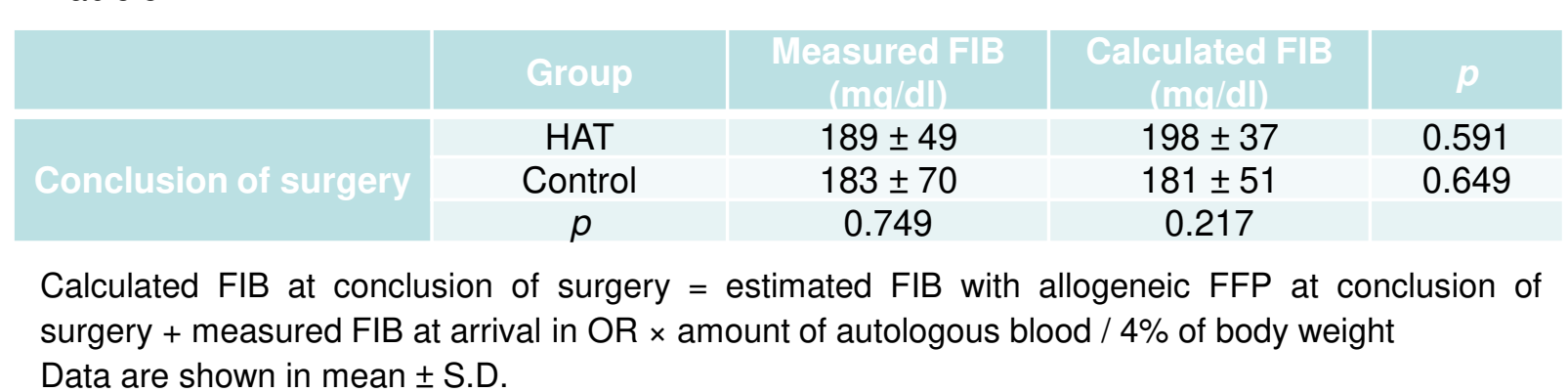

Conclusion: FIB estimation formula from Fuji-san classification gives good estimation of FIB in allogeneic blood transfusion cases but not in hemodilutional autologous blood transfusion cases. The use of Fuji-san classification formula is limited to allogeneic FFP transfusion and autologous blood should be calculated with fibrinogen amount and circulating blood amount estimation.

Reference: Kawashima S,et al. Four-Group Classification Based on Fibrinogen Level and Fibrin polymerization Associated with Postoperative Bleeding in Cardiac Surgery. Clin Appl Thromb Hemost. 2016; 22(7): 648-55 